\title{
Author Index for Volume 64
}

\begin{tabular}{|c|c|c|c|}
\hline Abdalla, MA & 655 & Chen, $\mathrm{X}$ & 563 \\
\hline Abdelfattah, MS & 271,729 & Chen, Y & 71 \\
\hline Abe, A & 197 & Cheng, G & \\
\hline Abe, $\mathrm{T}$ & 769 & Cheng, J & \\
\hline Affonso, R & 327 & Choi, S-S & \\
\hline Ahn, JS & 461,587 & Chu, M & 79,1 \\
\hline Alakurtti, S & 475 & Chudíčková, M & \\
\hline Alexander, DC & 79,111 & Cooper, MA & \\
\hline Alunda, JM & 475 & Corral, MJ & \\
\hline Amano, S-i & 703 & Costa, TD & \\
\hline An, M-M & 333 & Cullum, J & \\
\hline Ando, $\mathrm{K}$ & 599,613 & & \\
\hline Anzai, $\mathrm{H}$ & 221 & Dairi, T & \\
\hline Anzai, K & 613 & Dalgarno, DC & \\
\hline Aoki, Y & 607 & Damodaran, C & 1 \\
\hline Arakawa, K & 35 & Davies, JE & 7 \\
\hline Arima, S & 781 & de Araujo, BV & \\
\hline Armstrong, GS & 309 & de Pascale, G & \\
\hline Asai, $\mathrm{R}$ & 693 & Demain, $\mathrm{AL}$ & \\
\hline Asenjo, JA & 775 & Deng, M-R & \\
\hline Ashizawa, H & 289 & Ding, $Y$ & 339,56 \\
\hline & & Diniz, A & \\
\hline Baltz, RH & 79,111 & Djokic, L & \\
\hline Bamonte, $\mathrm{R}$ & 133 & Domingues, FC & \\
\hline Ban, Y-H & 155 & Donadio, $S$ & \\
\hline Bartl, F & 249 & Dong, L-J & \\
\hline Beese, $\mathrm{P}$ & 763 & Dorador, C & \\
\hline Beppu, T & 703 & $\mathrm{Du}, \mathrm{L}$ & \\
\hline Binh, CTT & 599 & Duan, $Y$ & 159,16 \\
\hline Boni-Mitake, M & 327 & Duangmal, K & 29 \\
\hline Boonlarppradab, C & 267 & Durán, $\mathrm{N}$ & \\
\hline Böttcher, C & 523 & & \\
\hline Brian, $\mathrm{P}$ & 79,111 & & \\
\hline Bringmann, $G$ & 229 & Eguchi, T & \\
\hline Brötz, E & 257 & Ekland, EH & \\
\hline Bruntner, C & 453 & El-Gamal, MI & \\
\hline Brzezinski, B & 249 & Endo, K & \\
\hline Buffé, C & 439 & Erhard, A & \\
\hline Bull, AT & 453,775 & Erkel, G & \\
\hline \multirow[t]{2}{*}{ Butler, MS } & 413 & & \\
\hline & & Falagas, ME & \\
\hline Cai, $\mathrm{P}$ & 167,533 & Fan, Y & \\
\hline Cai, S & 193 & Fawzi, M & \\
\hline Cai, Y & 559 & Feng, $\mathrm{X}$ & \\
\hline Cane, DE & 59,65 & Fidock, D & \\
\hline Carr, M & 649 & Fiebig, $\mathrm{HH}$ & \\
\hline Carver, KC & 159 & Fiedler, H-P & $229,257,453,595,7$ \\
\hline Casqueiro, J & 447 & Fitzgerald, JT & 759,7 \\
\hline Cavaletti, L & 133 & Flieger, M & \\
\hline Cazoto, LL & 395 & Floss, HG & \\
\hline Černý, J & 717 & Fujii, K & \\
\hline Cha, B-Y & 277 & Fujinuma, K & 5 \\
\hline Chang, Y & 735 & Fujita, J & \\
\hline Chávez, R & 447 & Fujita, K-i & 469,7 \\
\hline Chen, W-M & 571 & Fujita, Y & \\
\hline
\end{tabular}

$\begin{array}{lr}\text { Funabashi, M } & 495 \\ \text { Furihata, K } & 703 \\ \text { Fushinobu, S } & 65 \\ \text { Gange, AC } & \\ \text { Gao, F } & 427 \\ \text { Gao, L-y } & 317 \\ \text { Gao, P } & 17 \\ \text { Gebhardt, K } & 62 \\ \text { Glojnarić, I } & 22 \\ \text { Gong D D } & 51\end{array}$

Gong, D-L 459, 465

Gong, Y

167,673

Goodfellow, M 453, 595, 775

$\mathrm{Gu}, \mathrm{J}$

$\mathrm{Gu}, \mathrm{J}-\mathrm{Q} \quad 79,111$

$\mathrm{Gu}, \mathrm{Q}$

Guo, B-Q 571

Guo, J 345

Guo, Z 789

Haber, VE 515

Hachiya, A 551

Haltli, B 533

Hamada, M 289

Hamashima, H 547

Han, AR 155

Hang, DT 599

Hao, W 339

Harada, K-i 373

Harada, T 583

Haranaga, S 539

Harrison, PHM 483

Harvey, CJB 59

Hashimoto, J 399

Hashimoto, S 169, 697

Hayakawa, M 289, 399

Hayashi-Nishino, M 433

$\mathrm{He}, \mathrm{M}$

$\mathrm{He}, \mathrm{W}$

Helaly, S $\quad 775$

Helaly, SE $\quad 595$

Helmke, E 205

Hench, IB 717

Henrich, PP 799

Higa, F 539

Hino, M 169

Hirai, Y 741

Hirata, $\mathrm{H} \quad 373$

Hirohara, M 547

Hirose, T 303

Hirose, Y 117

Hong, Y-S 461

Horinouchi, S 93

Hosoe, T 211

Hranueli, D 89 


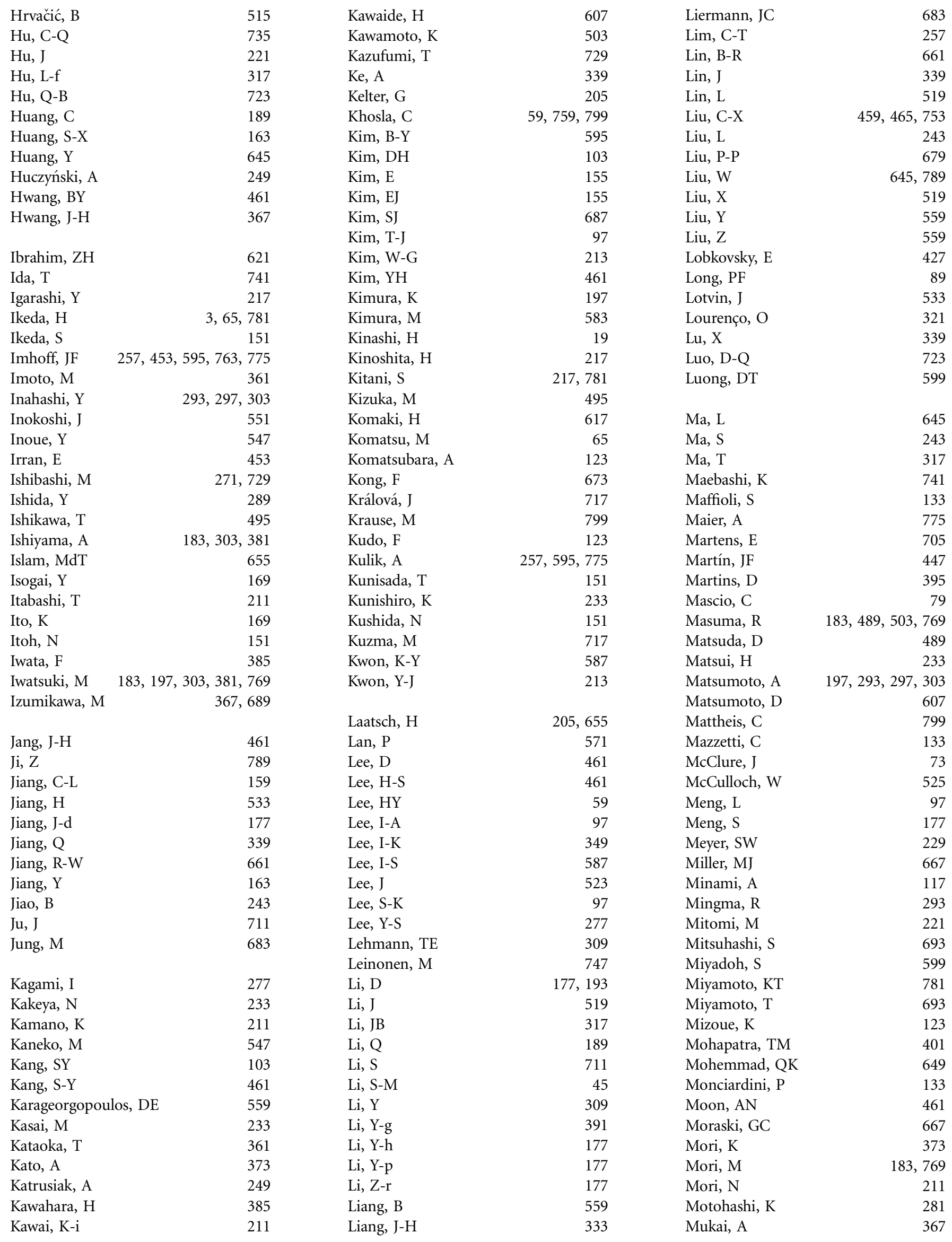




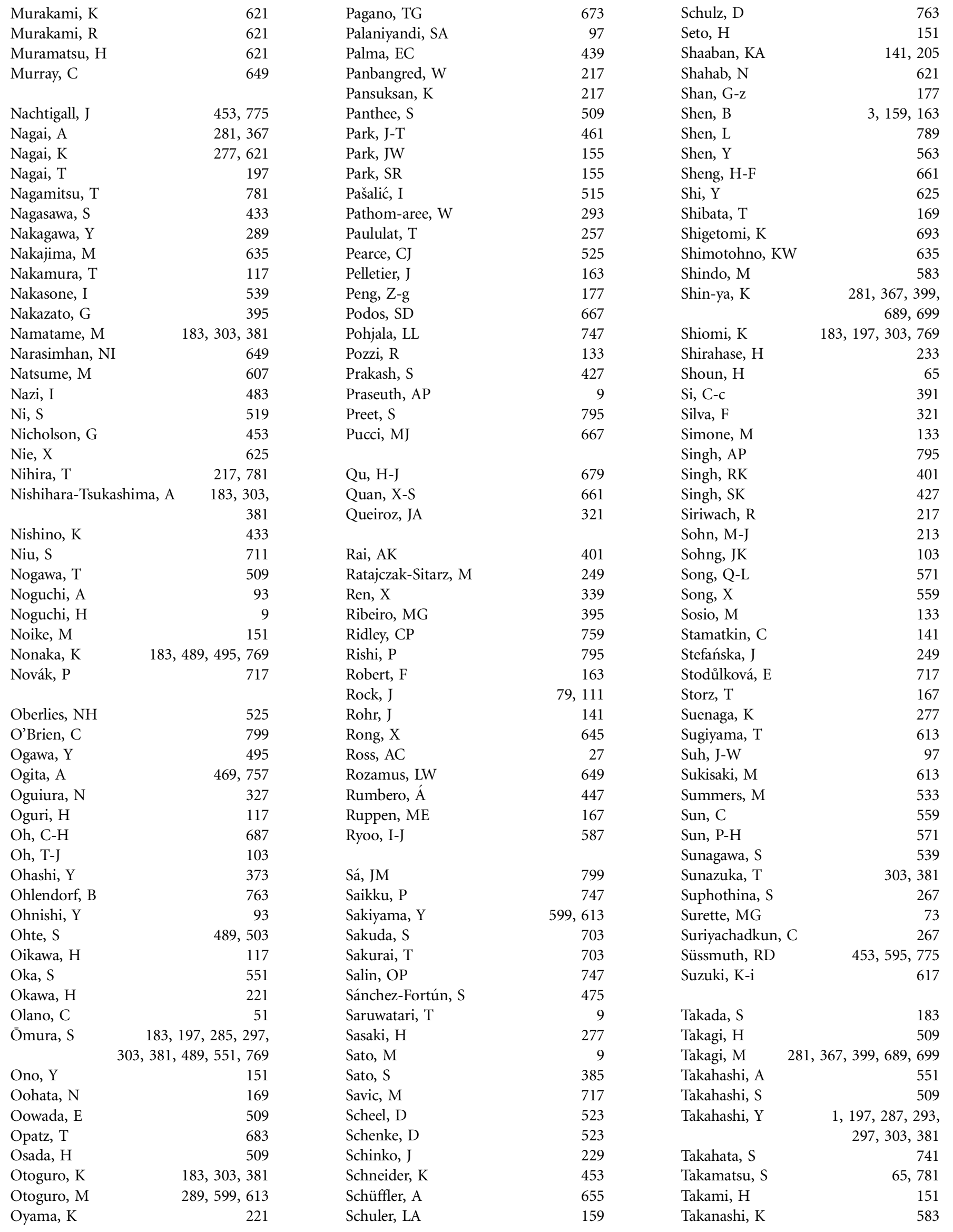


Takano, $\mathrm{H}$

Takatsu, T

Taketani, S

Tamaki, M

Tamura, $\mathrm{T}$

Tan, GYA

Tanaka, $\mathrm{H}$

Tanaka, N

Tanaka, R

Tanaka, S

Tanaka, T

Tao, L-m

Tashiro, E

Tateyama, M

Teruya, T

Thamchaipenet, A

Thanassi, JA

Tian, L

Tian, $\mathrm{X}$

Tiwari, SP

Tkalčević, VI

Tobwor, $\mathrm{P}$

Togashi, N

Tomoda, H

Torikai, K

Toume, K

Tsao, R

Ubukata, M

Uchida, M

Uchida, R

Uchida, Y

Ueda, J-y

Ueda, K

Ueda, S

Ullán, RV

Uno, T

Uramoto, $\mathrm{M}$

Usuki, Y

Vaca, I

Van Hop, D

VanderMolen, KM

Vasiljevic, B

Vederas, JC

Verma, VC

Vikineswary, S

von Tiedemann, A
Vuorela, HJ

Vuorela, PM

Wang, C

Wang, $\mathrm{F}$

Wang, $\mathrm{H}$

Wang, J-D

Wang, J-J

Wang, $\mathrm{R}$

Wang, X-J

Wang, $\mathrm{Y}$

Wang, Y-H

Wang, Y-Y

Watanabe, $\mathrm{K}$

Wei, G

Wert, L

Wiese, J

Willbold, S

Win, HY

Wong, L

Woo, J-T

Wright, GD

Wu, C-Z

$\mathrm{Wu}, \mathrm{L}$

Wu, W

Xiang, W-S

Xiang, Z-D

Xin, $\mathrm{H}$

$\mathrm{Xu}, \mathrm{L}-\mathrm{H}$

$\mathrm{Xu}, \mathrm{L}-\mathrm{Y}$

489, 503

583

367, 399

703

169

447

539

509

469, 757

447

599

525

717

27

427

257

655
$\mathrm{Xu}, \mathrm{M}-\mathrm{D}$

Yaguchi, T

Yamada, $\mathrm{H}$

Yamada, $\mathrm{K}$

Yamada, M

Yamada, S

Yamada, Y

Yamaguchi, A

Yamaguchi, T

Yamaki, H

Yamamoto, $\mathrm{K}$

Yamamoto, T

Yamamura, $\mathrm{H}$

Yamane, N

Yamasaki, S

Yamashita, M

Yamazaki, H
Yan, Y-J

Yang, SH 459

Yang, $\mathrm{X}$

Yang, X-L

Yao, G-W

Yao, X-S

Ye, Y

Yim, G

Yin, J

Yli-Kauhaluoma, J

Yonezawa, $\mathrm{T}$

Yoo, I-D

Yoo, YJ

Yoon, YJ

Yu, T-W

Yu, X

Yu, Z

Yun, B-S

Yutani, M

Zech, SG

Zeeck, A

Zhang, B-X

Zhang, C

Zhang, D-j

Zhang, G

Zhang, $\mathrm{H}$

Zhang, J

Zhang, L

Zhang, S

Zhang, W

Zhang, X-n

Zhang, Y

Zhao, B

Zhao, L-X

Zheng, J-K

Zheng, Z

Zhou, G-X

Zhu, H-H

Zhu, J

Zhu, T

Zhu, W-M

Zhu, X

Zhu, Y-l

Zhu, Z-X

Zinecker, $\mathrm{H}$

Zou, M

Zucko, J

243, 711

723

333

661

317

73

317

475

123, 277

587

155

155

35

559

159, 163

349

469, 757

649

229

753

711

391

327,711

$339,465,711,753$

$465,591,789$

243

189, 711, 723

571,711

317

723

193

159, 163

679

339

661

345

339

193, 673

679

563

317

459

433

169

503
763

625 